\title{
CHEP 2019: Preface to the Proceedings
}

\author{
Caterina Doglioni ${ }^{1, *}$, Paul Jackson ${ }^{2, * *}$, Waseem $\mathrm{Kamleh}^{2, * * *}$, Doris Y $\mathrm{Kim}^{3, * * * *}$, Lucia \\ Silvestris ${ }^{4 \dagger}$ and Graeme A Stewart ${ }^{5, *}$ \\ ${ }^{1}$ Fysiska institutionen, Lunds universitet, Lund, Sweden \\ ${ }^{2}$ Department of Physics, University of Adelaide, Adelaide, Australia \\ ${ }^{3}$ Soongsil University, Seoul 06978, South Korea \\ ${ }^{4}$ Istituto Nazionale Fisica Nucleare (INFN) Sezione di Bari, Via Orabona 4, 70125 Bari, Italy \\ ${ }^{5}$ CERN, 1 Esplanade des Particules, 1211 Geneva 23, Switzerland
}

\begin{abstract}
The $24^{\text {th }}$ International Conference on Computing in High Energy and Nuclear Physics (CHEP) took place at the Adelaide Convention Centre, Adelaide, South Australia from 4-8 November 2019. 525 registered participants took part in the conference, where there were plenary sessions as well as a wide ranging set of ten parallel tracks across all areas of work in the field and allied sciences. The conference hosted 34 plenary presentations, 370 oral presentations in parallel sessions and 131 posters.
\end{abstract}

\section{Foreword}

In November of 2019, the $24^{\text {th }}$ International Conference on Computing in High Energy and Nuclear Physics (CHEP) was held in Adelaide, South Australia. This signified the first iteration of the conference to be hosted in Australia.

Australia is, by just about anyone's measure of distance, a long way from everywhere else in the world. This is especially true with regards to the focal centres of the high energy and nuclear physics communities. Flying from Adelaide to CERN takes around 24 hours, with a similar time required to reach North America. Even travelling to relatively nearby Asia presents the challenge of sitting on a plane for 10 hours or more.

It was thus a great delight to welcome to Adelaide more than 540 people to participate in CHEP 2019, comprised of scientific delegates and accompanying persons. During the conference, the Adelaide weather of late spring granted long days of sunshine and a comfortable level of warmth, well before the more extreme heat of the Australian summer would commence. This yielded a pleasant atmosphere for delegates to enjoy the organised excursions, choosing to tour either the McLaren Vale wine region or Cleland Wildlife Park. After the completion of the conference, many overseas travellers visited the surrounding regions of South Australia, or journeyed interstate to explore other parts of Australia.

\footnotetext{
*e-mail: caterina.doglioni@ cern.ch

**e-mail: paul.douglas.jackson@ cern.ch

***e-mail: waseem.kamleh@adelaide.edu.au

****e-mail: dorisykim@ssu.ac.kr

†e-mail: lucia.silvestris@cern.ch

†e-mail: graeme.andrew.stewart@cern.ch
} 
Of course, at the time we did not know that for many this would be the last opportunity for international travel before world events would change everyone's lives. With the onset of the coronavirus pandemic, 2020 presented an entirely new set of challenges to all nations. The CHEP 2019 proceedings catalogue many diverse advances in knowledge, reminding us of the value that is generated by gathering in-person for a conference. This gives all the more reason to look forward to the next face-to-face meeting of the CHEP community in Norfolk, Virginia.

\section{CHEP conference series}

The CHEP conference series was established in 1985, and since then has been one of the most important events in the field of computing in high-energy and nuclear physics. The conference covers a broad set of topics such as online, offline and distributed computing; software development, simulation, reconstruction and analysis packages; data handling, data bases and storage solutions; clouds, virtualisation and containers; networking and facilities, including high performance computing. It provides a valuable discussion platform, enabling the exchange of ideas between physicists, computing scientists and software engineers, as well as between renowned experts and young researchers.

Focusing on the achievements, ongoing activities, plans, and trends in the field, the CHEP conference is held every 18 months. The host location is selected on a rotating basis between the geographical regions of Europe, the Americas, and Asia Pacific. The list of past CHEP conferences is shown in Table 1 .

Table 1: Dates and locations of previous CHEP conferences.

\begin{tabular}{|c|c|c|}
\hline Name & Dates & Location \\
\hline CHEP'85 & 25-28 June 1985 & Amsterdam, Netherlands \\
\hline CHEP'87 & 2-6 February 1987 & Asilomar, California, USA \\
\hline CHEP'89 & 10-14 April 1989 & Oxford, England, United Kingdom \\
\hline CHEP'90 & 9-13 April 1990 & Santa Fe, New Mexico, USA \\
\hline CHEP'91 & 11-15 March 1991 & Tsukuba, Japan \\
\hline CHEP'92 & 21-25 September 1992 & Annecy, France \\
\hline CHEP'94 & 21-27 April 1994 & San Francisco, California, USA \\
\hline CHEP'95 & 18-22 September 1995 & Rio de Janeiro, Brazil \\
\hline CHEP'97 & 7-11 April 1997 & Berlin, Germany \\
\hline CHEP'98 & 31 August-4 September 1998 & Chicago, Illinois, United States \\
\hline CHEP'2000 & 7-11 February 2000 & Padova, Italy \\
\hline CHEP'01 & 3-7 September 2001 & Beijing, China \\
\hline CHEP'03 & 24-28 March 2003 & San Diego, California, USA \\
\hline CHEP'04 & 27 September - 1 October 2004 & Interlaken, Switzerland \\
\hline CHEP'06 & $13-17$ February 2006 & Mumbai, India \\
\hline CHEP'07 & 2-7 September 2007 & Victoria, British Columbia, Canada \\
\hline CHEP'09 & 21-27 March 2009 & Prague, Czech Republic \\
\hline CHEP'10 & $18-22$ October 2010 & Taipei, Taiwan \\
\hline CHEP'12 & 21-25 May 2012 & New York, New York, USA \\
\hline CHEP'13 & 14-18 October 2013 & Amsterdam, Netherlands \\
\hline CHEP'15 & 13-17 April 2015 & Okinawa, Japan \\
\hline CHEP'16 & 10-14 October 2016 & San Francisco, California, USA \\
\hline CHEP'18 & 9-13 July 2018 & Sofia, Bulgaria \\
\hline
\end{tabular}




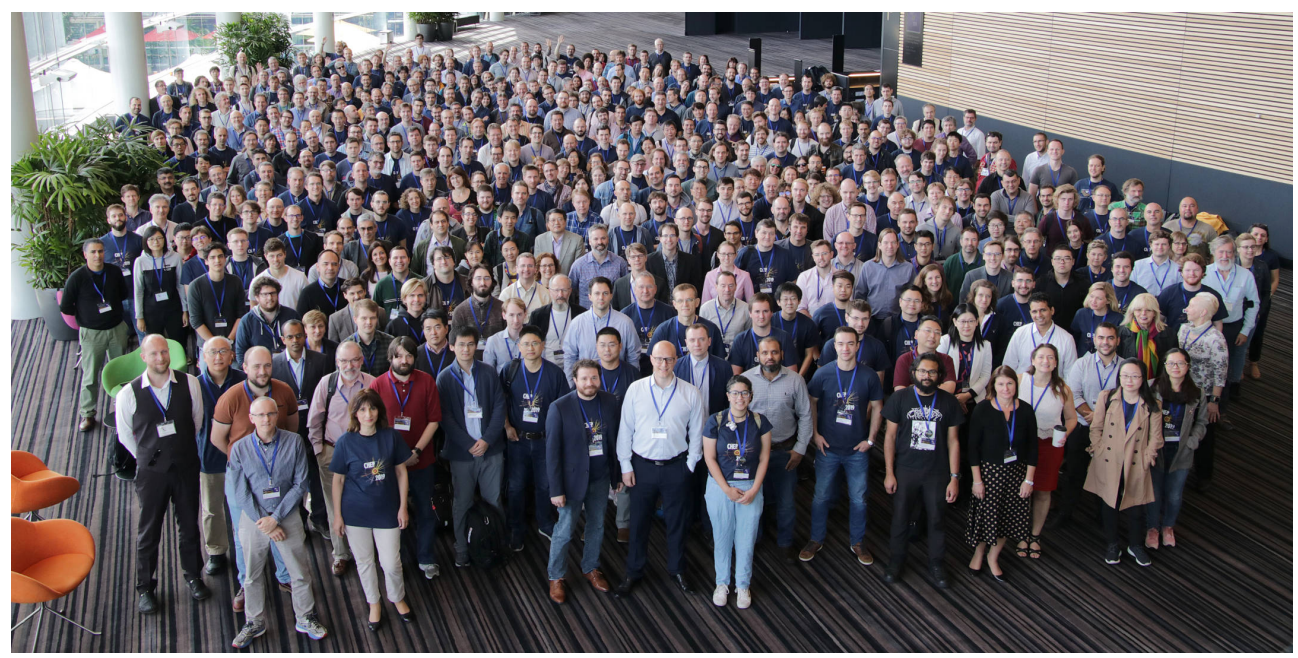

\section{CHEP 2019 Conference}

\subsection{Venue}

The CHEP 2019 conference was held at the Adelaide Convention Centre (pictured below).

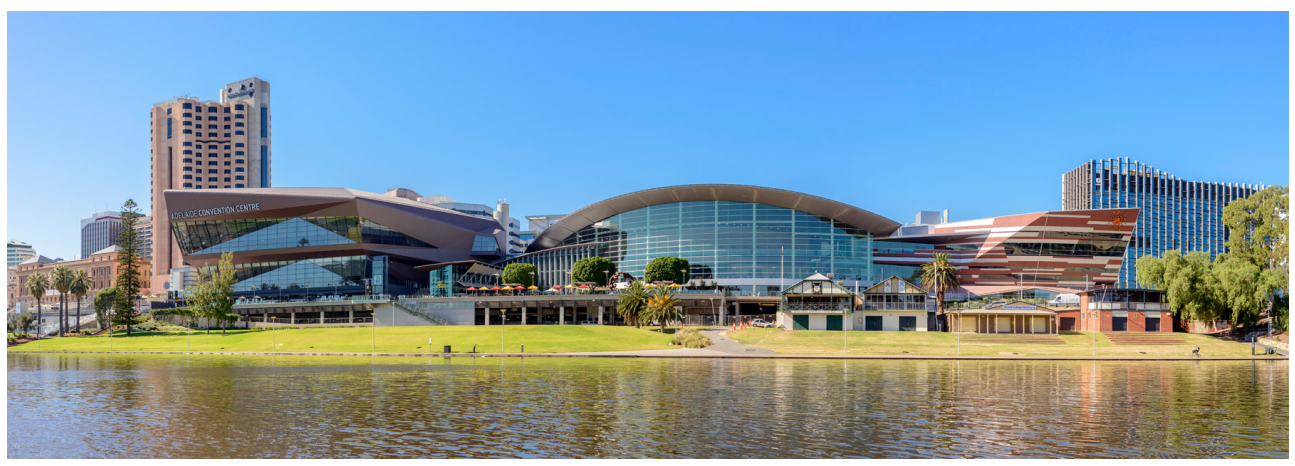

\subsection{Conference Programme}

The programme for CHEP 2019 consisted of plenary sessions and 10 parallel tracks. In the plenary sessions, 18 scientific keynote talks were given covering state-of-the-art developments in HEP (LHC Experiments, Belle II, DUNE, JUNO); other data intensive experiments and systems (SKA, gravitational waves, real-time alert systems, computational chemistry); and key technological and strategic areas (quantum computing, artificial intelligence, cyberthreats and security, European Strategy Update). In addition, two sessions were organised on diversity and inclusivity. There were 10 track highlight talks as well as a presentation from the organisers of the next face-to-face CHEP conference, which will be hosted in Norfolk, Virginia, USA.

In the parallel programme the keywords associated with Tracks 1-9 are shown in Table 2 . Track X, so named for being a cross-over track and the tenth parallel track, was specially constructed from papers that had themes of common interest between Tracks 1, 2 and 9. 370 oral presentations were given in the parallel sessions. 131 posters were presented with 2 dedicated poster sessions taking place. 
Table 2: CHEP 2019 Parallel Tracks

\begin{tabular}{|c|c|c|}
\hline & Name & Keywords \\
\hline 1 & $\begin{array}{l}\text { Online and } \\
\text { Real-time } \\
\text { Computing }\end{array}$ & $\begin{array}{l}\text { Data acquisition; high-level triggers; trigger-less ac- } \\
\text { quisition; online data calibration; online reconstruc- } \\
\text { tion; machine learning for online; real-time analysis; } \\
\text { event building; configuration and access controls; de- } \\
\text { tector control systems; real-time analytics and moni- } \\
\text { toring; heterogeneous resources online; trigger tech- } \\
\text { niques and algorithms; hardware trigger algorithms; on- } \\
\text { line databases }\end{array}$ \\
\hline 2 & $\begin{array}{l}\text { Offline } \\
\text { Computing }\end{array}$ & $\begin{array}{l}\text { Detector simulation; MC event generation; offline re- } \\
\text { construction; detector geometries; non-event data; data } \\
\text { classification; fast simulation; machine learning for of- } \\
\text { fline; offline databases }\end{array}$ \\
\hline 3 & $\begin{array}{l}\text { Middleware and } \\
\text { Distributed } \\
\text { Computing }\end{array}$ & $\begin{array}{l}\text { Grid middleware; monitoring and accounting frame- } \\
\text { works; security models and tools; distributed workload } \\
\text { management; heterogeneous resource brokering (such } \\
\text { as GPUs); federated authentication and authorisation in- } \\
\text { frastructures; middleware databases }\end{array}$ \\
\hline 4 & $\begin{array}{l}\text { Data } \\
\text { Organisation, } \\
\text { Management and } \\
\text { Access }\end{array}$ & $\begin{array}{l}\text { Storage management frameworks; data access proto- } \\
\text { cols; object, metadata and event store systems; content } \\
\text { delivery and caching; data analytics; machine learning } \\
\text { for DOMA; FAIR data principles }\end{array}$ \\
\hline 5 & $\begin{array}{l}\text { Software } \\
\text { Development }\end{array}$ & $\begin{array}{l}\text { Software frameworks; software management, contin- } \\
\text { uous integration; software building; testing and qual- } \\
\text { ity assurance; software distribution; programming tech- } \\
\text { niques and tools; coding for heterogeneous architec- } \\
\text { tures; integration of ML and other toolkits }\end{array}$ \\
\hline 6 & Physics Analy & $\begin{array}{l}\text { Analysis algorithms; object identification; object cali- } \\
\text { bration; machine learning for analysis; analysis preser- } \\
\text { vation; analysis workflows; lattice QCD; theory calcu- } \\
\text { lations }\end{array}$ \\
\hline 7 & $\begin{array}{l}\text { Facilities, Clouds } \\
\text { and Containers }\end{array}$ & $\begin{array}{l}\text { Cloud resources; virtual machines and container tech- } \\
\text { nologies; anything-as-a-service; private and commer- } \\
\text { cial clouds; dynamic provisioning; networking; com- } \\
\text { puting centre infrastructure; management and monitor- } \\
\text { ing; facility integration of heterogeneous resources }\end{array}$ \\
\hline 8 & $\begin{array}{l}\text { Collaboration, } \\
\text { Education, } \\
\text { Training and } \\
\text { Outreach }\end{array}$ & $\begin{array}{l}\text { Collaborative tools; outreach activities; training initia- } \\
\text { tives; open data for outreach; data preservation for col- } \\
\text { laboration; event displays; open science cloud initia- } \\
\text { tives }\end{array}$ \\
\hline 9 & Exascale Science & $\begin{array}{l}\text { HPC and supercomputers; algorithm scaling; comput- } \\
\text { ing models; exabyte; exaflop; compute accelerators; } \\
\text { generic algorithms; weak scaling; quantum computing; } \\
\text { massive scale machine learning }\end{array}$ \\
\hline $\mathrm{X}$ & $\begin{array}{l}\text { Crossover } \\
\text { sessions from } \\
\text { online, offline and } \\
\text { exascale }\end{array}$ & $\begin{array}{l}\text { Selected contributions that were identified as of com- } \\
\text { mon interest, touching topics between online, offline } \\
\text { and exascale computing (Tracks } 1,2 \text { and 9) }\end{array}$ \\
\hline
\end{tabular}




\subsection{Programme Committee}

The CHEP 2019 Programme Committee (PC) developed the parallel sessions with appointed track conveners, who provided their expertise to review the papers submitted to the conference. The plenary programme was defined by the conference and PC chairs, in consultation with the International Advisory Committee (IAC). The PC members are listed in Table 3

Table 3: CHEP 2019 Programme Committee

\begin{tabular}{ll}
\hline Name & Affiliation \\
\hline Alessandra Forti & Manchester \\
Brian Paul Bockelman & Morgridge Institute for Research \\
Caterina Doglioni (PC Co-chair) & Lund \\
Catherine Biscarat & CNRS/IN2P3 \\
Chiara Rovelli & INFN Roma \\
Christoph Wissing & DESY \\
Christopher Pinkenburg & BNL \\
Chunhua Li & LiaoNing Normal University \\
Clara Nellist & Göttingen \\
Dimitri Arkhipkin & BNL \\
Doris Kim (PC Co-chair) & Soongsil \\
Fabio Hernandez & CC-IN2P3 \\
Felice Pantaleo & CERN \\
Graeme A Stewart (PC Co-chair) & CERN \\
Ilya Komarov & DESY \\
Isabel Campos Plasencia & CSIC \\
James Letts & UCSD \\
Jennifer Ngadiuba & CERN \\
Juan Guzman & CSIRO \\
Lauren Tompkins & Stanford \\
Lucia Silvestris (PC Co-chair) & INFN Bari \\
Martin Ritter & LMU München \\
Marzena Lapka & CERN \\
Maurizio Pierini & CERN \\
Mihaela Gheata & Institute of Space Science \\
Oksana Shadura & Nebraska-Lincoln \\
Paul Laycock & BNL \\
Phiala Shanahan & MIT \\
Sang-Un Ahn & KISTI \\
Sofia Vallecorsa & CERN \\
Stefan Roiser & CERN \\
Steven Farrell & NERSC \\
Steven Schramm & Geneva \\
Teng Jian Khoo & Geneva \\
Tibor Šimko & CERN \\
Tigran Mkrtchyan & \\
Tomoe Kishimoto & \\
Wei Yang & TESY \\
Yavier Espinal & Su Nakahama Higuchi \\
\hline
\end{tabular}




\subsection{International Advisory Committee}

The CHEP 2019 International Advisory Committee provided essential strategic advice for the organisation of the event and the members of the IAC are listed in Table 4 The CHEP 2019 IAC met 12 times, including one meeting held after the conference itself.

Table 4: CHEP 2019 International Advisory Committee

\begin{tabular}{|c|c|}
\hline Name & Affiliation \\
\hline Alessandra Forti & Manchester \\
\hline Amber Boehnlein & JLab \\
\hline Andreas Wicenec & UWA/ICRAR \\
\hline Concezio Bozzi & INFN Ferrara \\
\hline David Britton & Glasgow \\
\hline David Groep & Nikhef \\
\hline Davide Costanzo & Sheffield \\
\hline Caterina Doglioni (PC Co-chair) & Lund \\
\hline Doris Kim (PC Co-chair) & Soongsil \\
\hline Elizabeth Sexton-Kennedy & FNAL \\
\hline Gang Chen & IHEP \\
\hline Ghita Rahal & CC-IN2P3 \\
\hline Gonzalo Merino & Wisconsin \\
\hline Gordon Watts & Washington \\
\hline Graeme A Stewart (PC Co-chair) & CERN \\
\hline Hannah Short & CERN \\
\hline Heather Gray & UC Berkeley/LBNL \\
\hline Ian Bird & CERN \\
\hline Ikuo Ueda & KEK \\
\hline Jerome Lauret & $\mathrm{BNL}$ \\
\hline Josep Flix & PIC/CIEMAT \\
\hline Julia Andreeva & CERN \\
\hline Latchezar Betev & CERN \\
\hline Lucia Silvestris (PC Co-chair) & INFN Bari \\
\hline Maarten Litmaath & CERN \\
\hline Marco Cattaneo & CERN \\
\hline Maria Girone & CERN \\
\hline Markus Klute & MIT \\
\hline Michel Jouvin & IN2P3 - Orsay \\
\hline Michel Vetterli & Simon Fraser/TRIUMF \\
\hline Minh Huynh & UWA/ICRAR-CSIRO \\
\hline Mohammad Al-Turany & GSI \\
\hline Niko Neufeld & CERN \\
\hline Oxana Smirnova & Lund \\
\hline Patrick Fuhrmann & DESY \\
\hline Peter Clarke & Edinburgh \\
\hline Peter Elmer & Princeton \\
\hline Peter Hristov & CERN \\
\hline Petya Vasileva & CERN \\
\hline Randall Sobie & Victoria \\
\hline Richard Mount & SLAC \\
\hline
\end{tabular}


Table 4: CHEP 2019 International Advisory Committee

\begin{tabular}{ll}
\hline Name & Affiliation \\
\hline Sang Un Ahn & KISTI \\
Simon Lin & Academia Sinica \\
Simone Campana & CERN \\
Takanori Hara & KEK \\
Tommaso Boccali & INFN Pisa \\
Torre Wenaus & BNL \\
Vasil Georgiev Vasilev & Princeton \\
Wahid Bhimji & NERSC/LBNL \\
Xiaomei Zhang & IHEP \\
\hline
\end{tabular}

\subsection{Local Organisation}

The CHEP 2019 conference was chaired by Paul Jackson and Waseem Kamleh. The chairs wish to thank the secretariat, Sharon Johnson and Silvana Santucci, for their tremendous work in ensuring the organisation of the conference ran smoothly. Valuable assistance was provided by the members of the local organising committee, listed in Table 5 .

Table 5: CHEP 2019 Local Organising Committee

\begin{tabular}{ll}
\hline Name & Affiliation \\
\hline Anthony Thomas & Adelaide \\
Anthony Williams & Adelaide \\
Derek Leinweber & Adelaide \\
Jacinda Ginges & UQ \\
James Zanotti & Adelaide \\
Martin Sevior & Melbourne \\
Martin White & Adelaide \\
Paul Jackson (co-chair) & Adelaide \\
Peter Skands & Monash \\
Ross Young & Adelaide \\
Waseem Kamleh (co-chair) & Adelaide \\
\hline
\end{tabular}

\subsection{Proceedings Reviewers}

In addition to the programme committee members, listed in Table 3, we are very grateful to many members of the HEP community who helped review the papers that were submitted to this issue of the proceedings. The full list of reviewers is: Alaettin Serhan Mete, Alessandra Forti, Alex Martyniuk, Amol Jaikar, Andrea Perrotta, Andrea Sciabà, Andreas Gellrich, Andrzej Boźek, Anna Sfyrla, Antonio Di Pilato, Antonio Pérez-Calero Yzquierdo, Archana Sharma, Aristeidis Fkiaras, Arturo Sánchez, Attila Krasznahorkay, Bokrae Jung, Brian Bockelman, Byungyun Kong, Carlos Lourenco, Caterina Doglioni, Catrin Bernius, Cecilia Uribe Estrada, Chiara Rovelli, Christian Voss, Christoph Wissing, Christophe Haen, Christopher Pinkenburg, Christos Leonidopoulos, Chunhua Li, Clara Nellist, Concezio Bozzi, Costin Grigoras, Daniel Hackett, David Bouvet, David Lange, David Rohr, Diego Davila Foyo, Diogo Castro, Dmitri Smirnov, Dimitri Arkhipkin, Doris Yangsoo Kim, Dorothea Vom Bruch, Edgar Fajardo, Eileen Kuehn, Enric Tejedor, Fabio Cossutti, 
Fabio Hernandez, Farrukh Kahn, Federico Stagni, Felice Pantaleo, Frank Meier, Frank Winklmeier, Geonmo Ryu, Ghita Rahal, Graeme A Stewart, Hadrien Grasland, Hannah Short, Igor Sfiligoi, Ikuo Ueda, Ilya Komarov, Imma Riu, Isabel Campos Plasencia, Jakob Blomer, James Letts, Jason Webb, Javier Montejo Berlingen, Jean-Roch Vlimant, Jennifer Ngadiuba, Jeny Teheran, Jeongheon Kim, Jesus Puerta Pelayo, Jin Kim, Joao Fernandes, Juan Guzman, Julia Andreeva, Justas Balcas, Kenneth Bloom, Kenyi Hurtado Anampa, Kilian Lieret, Kilian Schwarz, Kunihiro Nagano, Lauren Tompkins, Lea Morschel, Lucia Silvestris, Luisa Arrabito, Maarten Litmaath, Manuel Giffels, Marco Mascheroni, Markus Prim, Martin Ritter, María Acosta Flechas, Masahiko Saito, Matthew Barrett, Maurizio Pierini, Max Fischer, Maxim Potekhin, Mihaela Gheata, Nicola Hardi, Oksana Shadura, Oxana Smirnova, Patricia Conde Muino, Paul Laycock, Paul Nilsson, Peter Love, Phiala Shanahan, Philippe Charpentier, Piotr Traczyk, Rene Caspart, Riccardo Di Maria, Riccardo Maria Bianchi, Rizart Dona, Ross Young, Ryu Sawada, Sandro Wenzel, Sang Un Ahn, Sang-Ho Na, Sangwook Bae, Sebastian Lopienski, Sergey Padolski, Simon George, Sioni Paris Summers, Sofia Vallecorsa, Sonia Natale, Stefan Roiser, Stefan Wunsch, Stefania Xella, Steven Farrell, Steven Goldfarb, Steven Schramm, Stewart Martin-Haugh, Sviatoslav Bilokin, Sébastien Gadrat, Teng Jian Khoo, Thomas Hartmann, Tibor Šimko, Tigran Mkrtchyan, Tim Smith, Tomoe Kishimoto, Torre Wenaus, Vasil Georgiev Vasilev, Vyacheslav Krutelyov, Wahid Bhimji, Walter Lampl, Wei Yang, Xavier Espinal, Xavier Valls Pla, Yu Nakahama Higuchi, Yuji Kato, Ziheng Chen.

\section{Sponsors}

The CHEP 2019 conference was supported by Principal Partners the Adelaide Convention Bureau, Adelaide Convention Centre, and the Government of South Australia. The CHEP 2019 host institution is the University of Adelaide, in partnership with the Centre for the Subatomic Structure of Matter and the ARC Centre of Excellence for Particle Physics at the Terascale. Additional sponsorship was provided by Pawsey Supercomputing Centre, Micron Technology, E4 Computer Engineering, NCI Australia, and Data Direct Networks.

\section{Principal Partners}

\section{Adelaide}

\section{Partners}

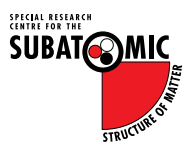

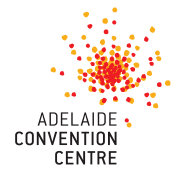

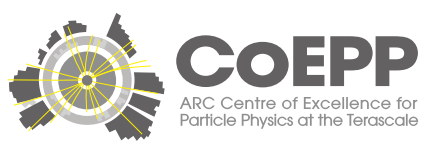

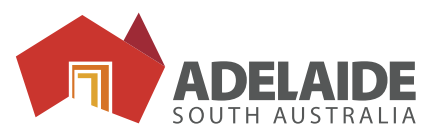

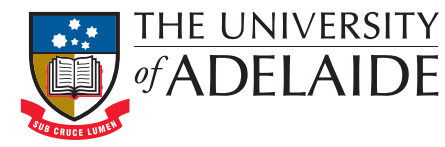

\section{Silver Sponsors}
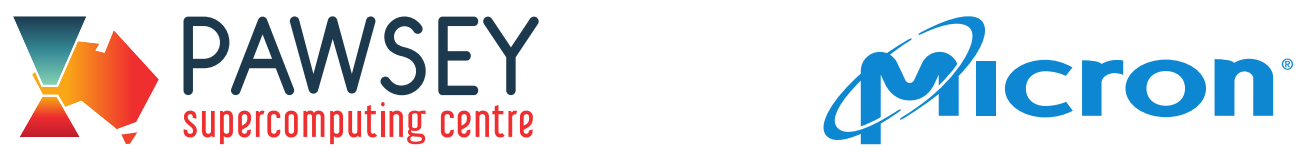

\section{Bronze Sponsors}
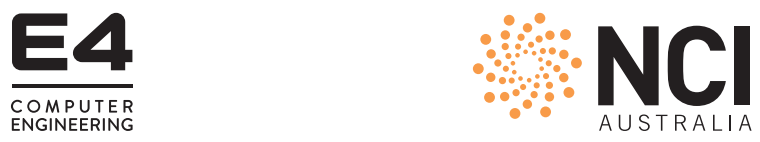